\title{
Biofouling as one of the Factors of Sustainable Development of Shipbuilding
}

\author{
Andrey Mosunov*, and Vladislav Evstigneev \\ Sevastopol State University, 33, Universitetskaya, Sevastopol, Russia
}

\begin{abstract}
Sea transportation is one of the cheapest and most widespread types of cargo transportation, and the process of fouling of the vessel's hull has an extremely negative impact on its maneuverability and running characteristics. This leads to excessive consumption of fuel, an increase in the cost of delivery of goods and environmental risks. Namely, when additional fuel is burned, the emission of the greenhouse gas $\mathrm{CO}_{2}$ increases. Considering the accompanying environmental risks in the longterm planning of the shipbuilding industry will ensure the sustainability of its development. In the study we suggest a simple way to assess quantitatively the development strategy of shipbuilding from the point of view of the accompanying economic and environmental consequences by constructing a matrix model for the industry development. The matrix model was applied to the Strategy for Development of the Shipbuilding Industry in Russia until 2035. In the future, on the basis of the offered model, it is possible to develop optimal environmentally neutral scenarios for shipbuilding development.
\end{abstract}

\section{Introduction}

Strategies for development of sectors of the economy are developed based on the expected needs of the state or business for the forecasted period of years. Often such strategies are aimed only at meeting increasing needs without detailed analysis of related environmental risks. Underestimation of such risks in the future may lead to an additional set of measures to counter the adverse consequences provoked by a given vector of the industry development. Against the background of the trends observed in the global climate system, the problem of mitigating the effects of climate change by reducing industrial greenhouse gas emissions (GHG) has gained additional focus [1]. Considering the commitments made by many countries to reduce GHG, strategies for development of economic sectors and industrial sectors shall consider the possible environmental and climatic risks within their implementation. In Russia, at the state level, a number of national initiatives have been adopted, enshrined in the relevant Strategies, Presidential Decrees and Government Orders.

Introduction of an approach to developing strategies not only for development, but also for managing the risks generated by this, will make it possible to get closer to implementation of "sustainable development" concept [2]. Sustainable development is about meeting the needs of the present generation without threatening future generations to

*Corresponding author: mosunovandrey@mail.ru 
meet their own needs. A measure of this development is preservation of the stability of physical and ecological systems achieved through preservation and rational use of natural resources.

One example of an industry in which a sustainable development strategy is necessary is shipbuilding. According to the International Maritime Organization (IMO), the merchant fleet provides up to $90 \%$ of the global transcontinental transportation of goods [3]. Shipbuilding has a multiple effect on development of related industries such as metallurgy, machine tool building, engine building, and etc. Russia has developed a Strategy for Development of the Shipbuilding Industry until 2035 that envisages the annual production of civil vessels and marine equipment with displacement of more than 80 tons [4]. However, strategies of this kind lack a prospective assessment of possible environmental risks associated with this development. Moreover, some of these risks are not obvious and are not considered as the main ones that shall be considered. The factor of marine fouling of ship hulls is among those risks.

Marine biofouling of ships and hydraulic structures is a global problem that has not yet been effectively resolved. The urgency of solving this problem is due, first of all, to the economic feasibility of operating vessels and offshore hydraulic structures. Namely, biofouling of the hulls of vessels leads to increase in the surface friction of the hull up to $70 \%$ [5] and decrease in its running performance up to $50 \%$ of its nominal value. A direct consequence is increase in fuel consumption that leads not only to increase in the cost of cargo delivery, but also to environmental consequences in the form of additional fuel combustion. This, in its turn, leads to increased $\mathrm{GHG} \mathrm{CO}_{2}$ emissions. Total GHG emissions associated with vessel transportation are considered to be about 930 million tons [6].

Controlling marine fouling has traditionally been associated with development of biocide-releasing coatings that is still the most reliable way to prevent biological fouling. However, after prohibition of environmentally toxic organometallic (lead-, mercury- and tin-containing) antifouling coatings, research and the search for new effective biocidal materials and principles for suppressing the vital activity of biofouling have become a significant scientific problem that still awaits its solution. Use of various nanocomposite materials is currently considered one of the most promising components of anti-fouling coatings [7].

In this paper, the goal was to develop a simple way to quantify the strategy for development of shipbuilding from the point of view of the accompanying economic and environmental consequences by constructing a matrix model of strategic planning for the industry.

\section{Materials and Methods}

The data for analysis were taken from the Strategy for Development of the Shipbuilding Industry until 2035, Appendix 1 "Target Indicators for Implementation of the Strategy", the item "The Volume of Production of Civil Vessels and Marine Equipment with Displacement of More than 80 tons for the Domestic Market and for Export on Accrual Basis from 2019" [4].

The data for the period of 2019-2035 were used to estimate the planned rates of vessel release, and to build a matrix model that includes both the planned release rates and considers the rate of fouling on the surface of vessels and excessive fuel consumption in relation to the nominal value. 


\section{Results and Discussion}

In our paper, we set the task of creating a matrix model of a strategic plan for development of the shipbuilding industry. The main prerequisites for building a matrix model are as follows:

1) discrete moments of time (years) are considered, during which one of the following events occurs:

- a certain number of new vessels are being built and put into operation in accordance with the Strategy;

- early commissioned vessels are moving from one type of surface condition to another, more "heavy" type in terms of the composition and severity of fouling and changes in running characteristics;

- in a certain year, the vessel is docked to carry out maintenance and repair work and restore running performance;

2) the standard duration of an interdock period of 5 years is considered, at the end of which works to clean the (usually mechanically) the hull from fouling are planned;

3 ) deterioration of the propulsive qualities of vessels with increase in their age is not considered;

4) the discrete (annual) inter-dock fouling cycle of the vessel is considered. According to the data of $[8,9]$, such a cycle and increase in power with increase in the roughness of the hull to maintain the speed of the vessel is presented as follows:

Stage 1 - primary fouling, microalgae; additional increase in power to overcome friction up to $10 \%$;

Stage 2 - formation of the dense mucous fouling film; increase in power up to $20 \%$;

Stage 3 - lime fouling leads to increase in power up to $35-80 \%$, depending on the degree of fouling "severity".

4 ) in the first rough approximation, we assume that the increment in power to maintain the speed of the vessel is proportional to the increment in fuel consumption. In its turn, the amount of additional $\mathrm{CO}_{2}$ emissions into the atmosphere is proportional to the amount of excessive fuel consumption.

5) as a target characteristic in modeling, the total increment of engine power, expressed in $\%$, can be used for all operating vessels. Such total increment will be proportional to the excess fuel consumption and carbon dioxide emissions into the atmosphere.

Therefore, it is assumed that the grouping of new vessels has some internal structure depending on the degree of fouling of the underwater part of the hull. Let us define an interdock period of 5 years, then the structure of the grouping of vessels contains 5 possible states. This detailing of the structure leads to the class of matrix models. Let at the initial moment of time the grouping be described by the column vector $X\left(t_{0}\right)=$ $\left(1, x_{1}\left(t_{0}\right), \ldots x_{N}\left(t_{0}\right)\right)^{T}$, where $x_{i}$ is the number of vessels in the $i$ th state of fouling, a single first element of the vector is necessary to introduce an external factor into the model commissioning the new vessels (see below). Vector $X\left(t_{1}\right)$ characterizing the state of the grouping of vessels at the next moment in time, for example, in a year, is associated with the vector $X\left(t_{0}\right)$ through the transition matrix $L$ :

$$
\boldsymbol{X}\left(t_{1}\right)=\boldsymbol{L} \boldsymbol{X}\left(t_{0}\right)
$$

Let us establish the form of this matrix. The first type of vessels is obtained considering two processes. The first is the commissioning new vessels in accordance with the Strategy, the second is the return to service of vessels after docking:

$$
x_{1}\left(t_{i}\right)=F\{i\}+\left(1-b_{1}\right) x_{1}\left(t_{0}\right)+b_{5} x_{5}\left(t_{0}\right) .
$$


$F\{i\}$ plays the role of a certain strategy operator associated with the current year $i$; it can be either a tabulated function with specific planned indicators, or an analytical function (for example, a linear one) that determines the trend in development of shipbuilding volumes. The second term reflects the fact that due to the high-quality coating on the hull and the anti-fouling compound used, some of the vessels $\left(1-b_{1}\right)$ may remain in the first most effective state, while the other fraction of $b_{1}$ vessels will go into a worse second state. The equation also assumes that all vessels in a state of 5 years of operation and corresponding fouling are sent for docking, their return to service is equivalent to contribution to the first group of vessels.

The second group of vessels at time $i$ is determined by transition to this state of vessels from the first group and the probability that some of the vessels of the second group will retain their state in a given year:

$$
x_{2}\left(t_{i}\right)=b_{1} x_{1}\left(t_{0}\right)+\left(1-b_{2}\right) x_{2}\left(t_{0}\right) .
$$

Further, all subsequent groups of states are described similarly. It shall be noted that the factors in the equations in the general case $b_{i} \in[0,1]$. Based on the equations written out, we construct the transition matrix $\boldsymbol{L}$ for 5 states of the grouping of vessels:

$$
\boldsymbol{L}=\left(\begin{array}{cccccc}
F\{i\} & 0 & 0 & 0 & 0 & 0 \\
F\{i\} & 1-b_{1} & 0 & 0 & 0 & b_{5} \\
0 & b_{1} & 1-b_{2} & 0 & 0 & 0 \\
0 & 0 & b_{2} & 1-b_{3} & 0 & 0 \\
0 & 0 & 0 & b_{3} & 1-b_{4} & 0 \\
0 & 0 & 0 & 0 & b_{4} & 1-b_{5}
\end{array}\right) .
$$

Therefore, knowing the structure of the matrix $\boldsymbol{L}$ and the initial state of the grouping the column vector $\boldsymbol{X}\left(t_{0}\right)$ - you can predict the state of the grouping at any given moment in time: $\boldsymbol{X}\left(t_{i}\right)=\boldsymbol{L} \boldsymbol{X}\left(t_{i-1}\right)=\boldsymbol{L} \boldsymbol{L} \boldsymbol{X}\left(t_{i-2}\right) \ldots=\boldsymbol{L}^{\boldsymbol{i}} \boldsymbol{X}\left(t_{0}\right)$. Knowing the structure of the grouping in a specific forecast year, it is possible to calculate the total excess of fuel consumption due to fouling, for example, using information from the 4th prerequisite (see above). The excess consumption is expressed as a percentage of the nominal fuel consumption, multiplying by which, the excess consumption (or additional $\mathrm{CO}_{2}$ emissions) can be obtained in absolute terms.

Let us show the result of applying this model using the example of the Strategy for Development of the Shipbuilding Industry in Russia until 2035. According to the Strategy [4], it is planned to build new vessels in Russia with a cumulative total of 358 units by 2035. However, the rate of new vessels construction decreases annually (Fig. 1). According to the estimate of the linear trend in Fig. 1 such a decrease in the rate of construction by one unit per year. Let's introduce this regularity inherent in the Strategy into the model. Let's assume that the initial conditions for 2019 are commissioning 30 vessels. Further, it is planned to annually reduce the number of new vessels by 1 unit. 


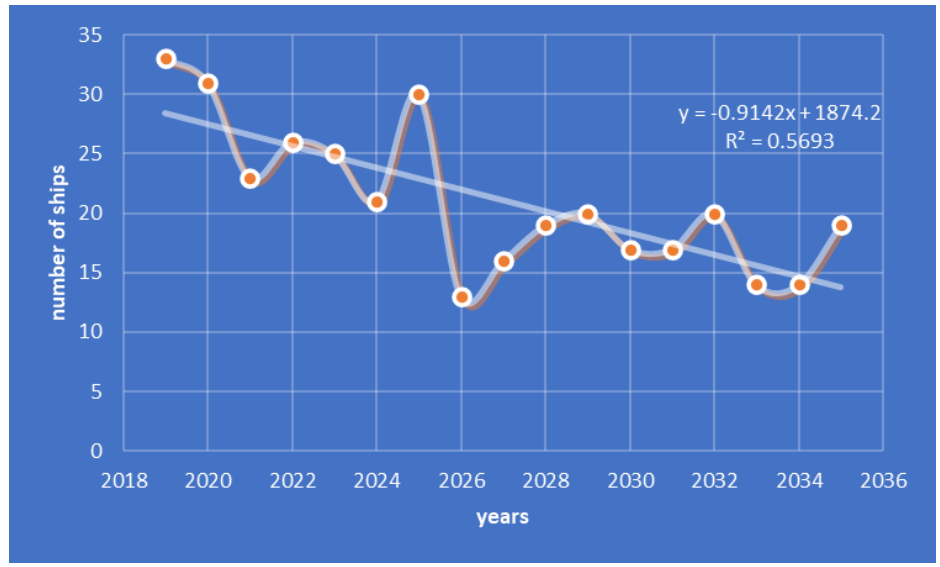

Fig. 1. Planned annual volume of vessel production until 2035 according to the Strategy [4].

To introduce this regularity into the model, we transform the transition matrix $\boldsymbol{L}$ and the column vector $\boldsymbol{X}$ so as to include the linear decrease in the number of vessels introduced into the matrix model:

$$
\boldsymbol{X}\left(\boldsymbol{t}_{\mathbf{0}}\right)=\left(\begin{array}{c}
30 \\
-1 \\
0 \\
0 \\
0 \\
0 \\
0
\end{array}\right) ; \quad \boldsymbol{L}=\left(\begin{array}{ccccccc}
1 & 1 & 0 & 0 & 0 & 0 & 0 \\
0 & 1 & 0 & 0 & 0 & 0 & 0 \\
1 & 0 & 1-b_{1} & 0 & 0 & 0 & b_{5} \\
0 & 0 & b_{1} & 1-b_{2} & 0 & 0 & 0 \\
0 & 0 & 0 & b_{2} & 1-b_{3} & 0 & 0 \\
0 & 0 & 0 & 0 & b_{3} & 1-b_{4} & 0 \\
0 & 0 & 0 & 0 & 0 & b_{4} & 1-b_{5}
\end{array}\right)
$$

The first two elements of the initial vector $\boldsymbol{X}\left(t_{0}\right)$ contain information about the strategy for introducing new ships, the transition matrix $\boldsymbol{L}$ is also adapted to this pattern. To calculate the excessive fuel consumption (and proportionally to GHG emissions), the following scenario was adopted: 1 year $-0 \%, 2$ years $-10 \%, 3$ years $-20 \%$, 4 years $35 \%, 5$ years $-50 \%$.

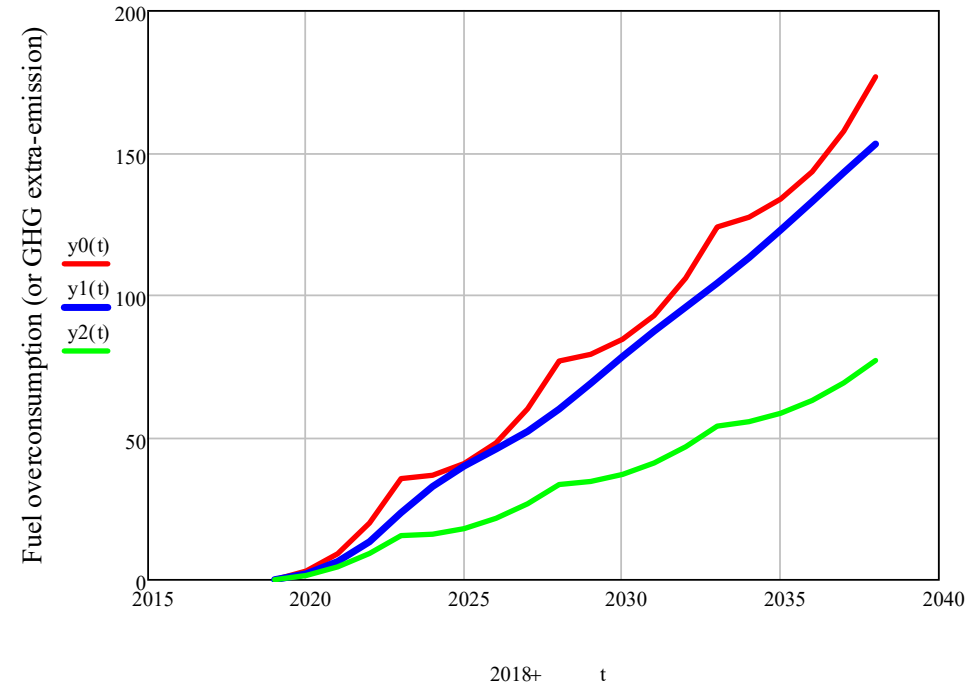

Fig. 2. Annual excess fuel consumption or additional GHG emissions due to fouling of the vessel's hulls according to the Strategy [4]. The values are provided in conventional units. The red line under the condition $b_{i}=1$, blue - under the condition $b_{i}<1$, green - with $b_{i}=1$ and when using antifouling coatings. 
The results of calculations using the matrix model are shown in Fig. 2 in 3 versions. In the first option (red line), the probability of transition of all ships from one state to another is equal to one. In this case, the graph shows periodic peaks in the excessive consumption of fuel and GHG emissions associated with the simultaneous deterioration of the fouling condition on all vessels, but after docking the values slightly decrease. In the second option (blue curve), it is assumed that a certain proportion of vessels may remain in the same state $\left(b_{i}<1\right)$. It can be seen that in this case the curve becomes substantially smoothed. The third option shows the effect of use of innovative anti-fouling coatings that reduce the loss of the vessel's course and, as a result, excessive fuel consumption. The scenario of excessive fuel consumption depending on the year was adjusted as follows: 1 year $-0 \%, 2$ years $-5 \%$, 3 years $-10 \%$, 4 years $-15 \%, 5$ years $-20 \%$. The general conclusion follows from the results that the increment in fuel consumption will grow steadily even with a decrease in the dynamics of launching new vessels.

\section{Conclusions}

The paper offers the matrix model for strategic planning of the shipbuilding industry that allows a rough estimate of the accompanying economic and environmental risks caused by fouling of the vessel's hull within its operation. On the basis of the offered model, it is possible to develop optimal environmentally neutral scenarios for construction of new ships, the choice of docking periods in combination with scenarios for introduction of innovative antifouling coatings. The indicator of optimality is the minimum of the total excessive consumption of fuel and GHG emissions. The matrix model is simplified and requires further development to increase its applicability in real practice.

\section{Acknowledgments}

The study was funded by Sevastopol State University (project № 47/06-31 "Biologically active bicomponent metal oxide nanoparticles as the basis for alternative compositions of antifouling coatings for ships and hydraulic structures").

\section{References}

1. R. K. Pachauri, L. A. Meyer, IPCC. Climate Change 2014: Synthesis Report. Contribution of Working Groups I, II and III to the Fifth Assessment Report of the Intergovernmental Panel on Climate Change (2014)

2. Take Action for the Sustainable Development Goals, https://www.un.org/

3. C. Hellio, D. Yebra, Advances in Marine Antifouling Coatings and Technologies (2009)

4. Strategy for the development of the shipbuilding industry until 2035, http://government.ru

5. B. S. Galil, C. McKenzie, S. Bailey, M. Campbell, I. Davidson, L. Drake, C. Hewitt, A. Occhipinti-Ambrogi, R. Piola, ICES Viewpoint background document: Evaluating and mitigating introduction of marine non-native species via vessel bio-fouling (2019)

6. N. Olmer, B. Comer, B. Roy, X. Mao, D. Rutherford, Greenhouse gas emissions from global shipping, 2013-2015 (2017)

7. D. Inbakandan, et al., Colloids Surf., B. 111, 636 (2013)

8. M. P. Schultz, Biofouling, 23, 331 (2007) 
9. M. P. Schultz, J. M.Walker, C. N. Steppe, K. A. Flack, Biofouling, 31, 759 (2015) 